\title{
COMENTARIO
}

\section{Algunas lecciones de las pasadas elecciones federales en México}

\author{
Clara Jusidman Rapoport
}

$\mathrm{L}$ as ponencias presentadas en el Seminario "Un balance de las elecciones de 2012", de la Cátedra "Jorge Alonso" del Centro de Investigaciones y Estudios Superiores en Antropología Social, publicadas en este número de la revista Desacatos, fueron formuladas por un grupo de académicos y especialistas que desde hace varios años dan seguimiento a las elecciones en el país, que además han realizado un activismo cívico y editorial comprometido con la democracia en el país. Los textos muestran con claridad sus preocupaciones centrales y ayudan a entender mejor la complejidad subyacente en los procesos electorales y en las relaciones de los actores participantes.

La evaluación de los ciudadanos respecto de la calidad del Proceso Electoral Federal 2011-2012 difiere ampliamente. Como señala Alberto Aziz Nassif en su texto, la percepción de un sector importante de la población es que la contienda no fue equitativa y estuvo plagada de intervenciones interesadas y de violaciones a la legislación, frente a las cuales la institucionalidad electoral no fue capaz de dar certidumbre y confianza. Algunos estudiosos del tema advierten crecientes dificultades para desarrollar reglas y herramientas que permitan contener y sancionar esas intervenciones ilegales y violaciones, pues se realizan en espacios privados con total opacidad y con la complicidad característica de los niveles de corrupción e impunidad que padecemos. No obstante la diferencia de 3.3 millones de votos $-6.62 \%$ entre el primero y segundo lugares para la presidencia de la república y la participación de $63.3 \%$ de la lista nominal de electores - siete puntos porcentuales por encima de la registrada en 2006-, muchas personas, no sólo las que apoyaron a los candidatos perdedores, quedaron con la impresión de que la contienda fue desigual. Además, consideran que esa desigualdad se fue construyendo desde antes del inicio formal del proceso electoral en octubre del 2011.

No existe un consenso sobre lo ocurrido aun cuando los procesos básicos de organización y calificación de la elección se cumplieron con apego a la norma. Se instalaron las más de 143000 casillas en todo el país y se integraron debidamente las mesas de funcionarios electorales, se distribuyeron los tiempos en medios con apego a la ley y se realizó un monitoreo adecuado de éstos con los límites que marca el Código Federal de Instituciones y Procedimientos

Some Lessons from the Last Federal Elections in Mexico

Clara Jusidman Rapoport: Iniciativa Ciudadana y Desarrollo Social, A. C., Distrito Federal, México cjusidman@yahoo.com.mx

Desacatos, núm. 42, mayo-agosto 2013, pp. 121-127 


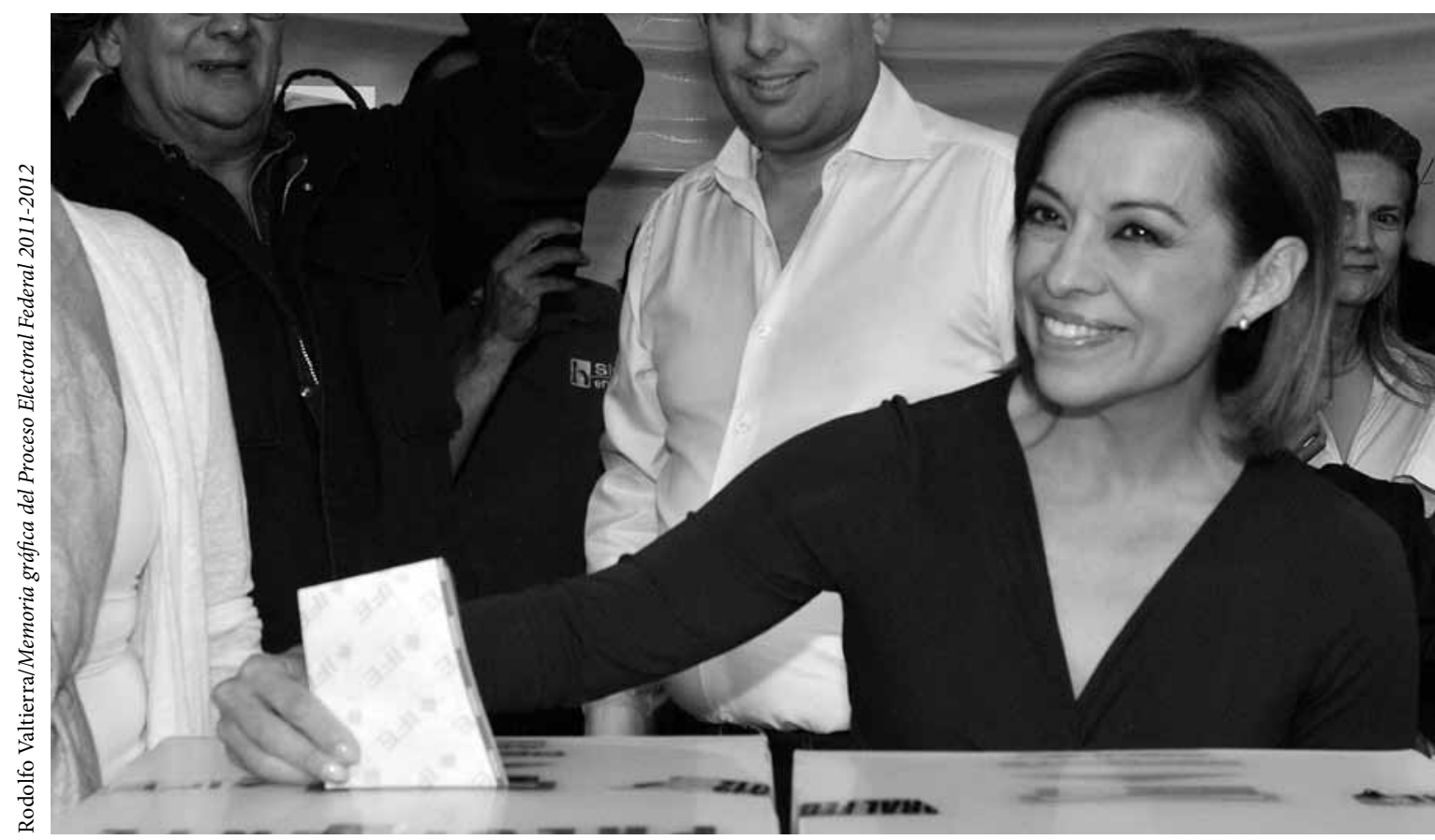

La candidata presidencial Josefina Vázquez Mota votando, 2012.

Electorales (Cofipe), se realizaron los debates previstos entre los candidatos, se llevó a cabo un amplio recuento de votos $-54.8 \%$ - mediante un trabajo intensivo y comprometido de los consejos distritales en un plazo muy corto, los procedimientos para tener cifras oportunas de los resultados - como el conteo rápido y el Programa de Rusultados Electorales Preliminares (PREP) - se cumplieron sin problemas, se resolvieron múltiples procedimientos especiales sancionadores, el Tribunal Electoral del Poder Judicial de la Federación (Trife) calificó y declaró válida la elección y otorgó las constancias de mayoría a los candidatos ganadores, etcétera.

Sin embargo, el control de varios de los factores, que desde la perspectiva de muchas personas no permiten considerarla como una elección equitativa, está fuera de las atribuciones, posibilidades y tiempos de intervención de la institucionalidad electoral actual. En cada elección los partidos políticos ponen en práctica viejas estrategias, como es el clientelismo o la recepción encubierta de recursos privados para desequilibrar la contienda, y surgen nuevas modalidades, en esta ocasión, la construcción mediática de candidatos con mucha anticipación o el uso de las encuestas como propaganda. Alberto Aziz Nassif lo señala claramente en su texto y Ana Mercedes Saiz Valenzuela lo menciona de algún modo: se trata de un ciclo en el que se busca prevenir la repetición de situaciones que desequilibraron la contienda electoral previa mediante reformas a la legislación electoral, se fortalecen los instrumentos y la institucionalidad, pero en cada elección aparecen otras modalidades para obtener ventajas y se hace necesario reformar de nuevo la legislación electoral. José Antonio Crespo considera que esa búsqueda de los partidos políticos y los candidatos para obtener ventajas sobre sus competidores forma parte de los procesos democráticos y que se observa en muchos otros países.

Aziz apunta que en cada elección se pone en disputa el modelo de reglas electorales con las que se compite. Se trata, dice, de ciclos complicados que inician 
con elecciones que no terminan por resolverse en las instancias institucionales, que generan conflictos y luego llegan reformas para modificar las reglas y corregir los excesos y los vacíos legales. Si bien es cierto que estos ciclos repetidos de elecciones-reforma legislativa-adecuación de las instituciones han contribuido a una mejoría sostenida de las reglas, procedimientos e instrumentos para lograr elecciones competidas, equitativas y confiables, es evidente que la organización de elecciones en México es cada vez más costosa tanto por los recursos legales como por los ilegales y que muchas de las dificultades que se observan en los procesos electorales tienen que ver más con el contexto general del país. En este sentido México enfrenta una corrupción generalizada, la ausencia del imperio de la ley, la creciente captura de espacios del Estado y de territorios por poderes de hecho, destacadamente el crimen organizado, la opacidad en el uso de recursos públicos, en especial por los gobiernos locales, y la persistencia de omisiones importantes de legislación en materia de telecomunicaciones, partidos políticos y lavado de dinero, por ejemplo. En tal contexto, deberíamos apreciar que el proceso electoral reciente pudo llevarse a cabo y fue posible realizarlo sin tropiezos graves en materia de violencia. Finalmente, afirma Aziz, la elección fue legal, pero no legítima -entendida ésta como la apropiación social de los procesos-. El autor sostiene que los procesos electorales llevan a un distanciamiento entre legalidad y legitimidad, y analiza las paradojas que afectaron la calidad y la legitimidad del proceso electoral reciente.

Lo que parece evidente en varios de los textos de este número de Desacatos es que los cambios legales e institucionales y los procesos electorales mismos se alejan cada vez más de los intereses de los ciudadanos y de la protección, el respeto y la garantía de sus derechos políticos. Saiz Valenzuela destaca que la reforma política de 2007-2008 disminuyó y acotó las posibilidades de intervención de los consejeros electorales ciudadanos en comisiones del Instituto Federal Electoral (IFE) y permitió la participación de los partidos políticos en las mismas, les quitó también la Unidad de Fiscalización de los Recursos de los Partidos Políticos al otorgarle autonomía técnica y de gestión o al designar el Congreso al contralor interno del Instituto, además de que sometió a los consejeros locales y distritales a la Ley Federal de Responsabilidades de los Servidores Públicos.

Desde mi punto de vista, los consejeros ciudadanos en varios niveles de la estructura deben dedicar ahora gran cantidad de tiempo para dirimir los conflictos entre los partidos y los candidatos al tener que resolver los llamados "procedimientos especiales sancionadores" en detrimento del tiempo que destinan a participar y dar seguimiento a la organización de las elecciones en todo el territorio nacional. Si anteriormente eran pocos los consejeros electorales del Consejo General que recorrían el país y los distritos electorales para constatar lo que efectivamente sucedía en el terreno, el agobio que ahora tuvieron para resolver los conflictos entre partidos políticos y confrontar la presión ejercida sobre ellos por representantes de los medios de comunicación los alejó más del contacto con toda la estructura ciudadana y profesional del IFE, que es la que realmente organiza todo el proceso. A la larga, esto se convierte en un problema de pérdida de integración y sentido de cuerpo de los ciudadanos y funcionarios electorales respecto de la institución, pues en muchas ocasiones tienen que afrontar circunstancias difíciles e incluso riesgosas ante amenazas de los representantes de los partidos políticos y de los poderes de hecho locales, así como conflictos entre los propios consejeros ciudadanos y los miembros del servicio profesional electoral.

En el mismo sentido de la creciente desvinculación de la legislación electoral con la protección de los derechos de los ciudadanos, Saiz Valenzuela realiza un análisis detallado con varios ejemplos para probar que las disposiciones incluidas en la mencionada reforma electoral en relación con el recuento de votos, asunto nodal en el conflicto postelectoral de 2006, no se hicieron para dar seguridad a los ciudadanos de que sus votos se contaron y que se contaron bien. De nuevo se trata de disposiciones 
para que los partidos políticos y los candidatos puedan controvertir los resultados de casillas específicas o para obligar a recuentos en los que, una vez ratificados los resultados originales, proceden a buscar la eliminación de casillas con el argumento de que hubo deficiencias, con el propósito último de cambiar los resultados de las votaciones, todo ello a costa de invalidar votos de los ciudadanos. Es decir, los procesos electorales están cada día más secuestrados por las cúpulas de los partidos políticos y las elites económicas y sociales, pues estos actores se distribuyen las candidaturas al Congreso y a los poderes ejecutivos, ejercen presión y con frecuencia intervienen en las decisiones de las instituciones electorales y se esfuerzan por inducir el voto mediante presiones.

Los ciudadanos, en cambio, poco o nada participan en la selección de los candidatos de los partidos, ni siquiera como miembros activos de éstos, y no tienen derecho a conocer los currícula de esos candidatos para ejercer un voto informado, como lo Comité Conciudadano para la Observación Electoral (Incide Social, 2006; CCOE e Incide Social, 2011: 119 y 158). Además, padecen las campañas electorales con incontables spots y no se atiende su exigencia de un mayor número de debates para conocer las posturas de los candidatos. Aquellos ciudadanos que tienen posiciones subordinadas por su trabajo o sus ingresos son sujetos de presión para decidir por quién votar. Todos enfrentan el riesgo de que sus votos sean invalidados por las disputas entre partidos o candidatos y terminan siendo gobernados $y$ representados por integrantes de las elites y de los poderes de hecho, que se encargan de gobernar y legislar con sus intereses particulares por encima de los colectivos.

El hartazgo que esta situación ha generado en la población fue retomado por los jóvenes universitarios de \#YoSoy132. El artículo de Jorge Alonso expone con claridad y detalle las razones del surgimiento del movimiento, el contenido y el origen de sus demandas, su expansión y articulación en todo el territorio

y con otros movimientos sociales, sus formas alternativas de organización, sus mecanismos pacíficos, lúdicos y creativos de protesta y las estrategias para enfrentar la represión. A pesar de ser un movimiento de jóvenes rechazan centralmente las formas de hacer política y ejercer el poder de las administraciones priistas. Por tanto, enfocaron su protesta contra el candidato presidencial de ese partido y contra los consorcios televisivos que obstaculizan la democratización de los medios de comunicación, es decir, lo que Jesús Cantú llama "la pluralidad interna y externa de medios".

\section{LA FALTA DE EQUIDAD EN LA CONTIENDA ELECTORAL}

Ahora bien, parece ser que son cuatro las razones esgrimidas para considerar que no fue una elección equitativa: primero, la estrategia y los recursos puestos en práctica desde 2006 por los consorcios televisivos para colocar en el imaginario de los militantes del Partido Revolucionario Institucional (PRI) y de toda la colectividad que sólo había un candidato viable para ser presidente, es decir, la construcción mediática de un candidato; en segundo lugar, una cantidad de recursos aportados por los partidos y sus aliados a las campañas políticas que rebasaron los límites establecidos por ley y, dentro de ello, la incorporación de recursos de fuentes privadas y de gobiernos locales en apoyo a las campañas; en tercer lugar, prácticas compartidas por todos los partidos de compra de votos e inducción de votantes, y finalmente, el papel jugado por las empresas encuestadoras, que más que informar contribuyeron a orientar los votos.

Las evidencias aportadas a las instituciones electorales sobre diversos incidentes registrados durante el proceso y detectados por los mismos consejeros distritales del IFE, por los observadores ciudadanos nacionales e internacionales, por los diversos partidos y por jóvenes del Movimiento \#YoSoy132 no fueron lo suficientemente contundentes como para 


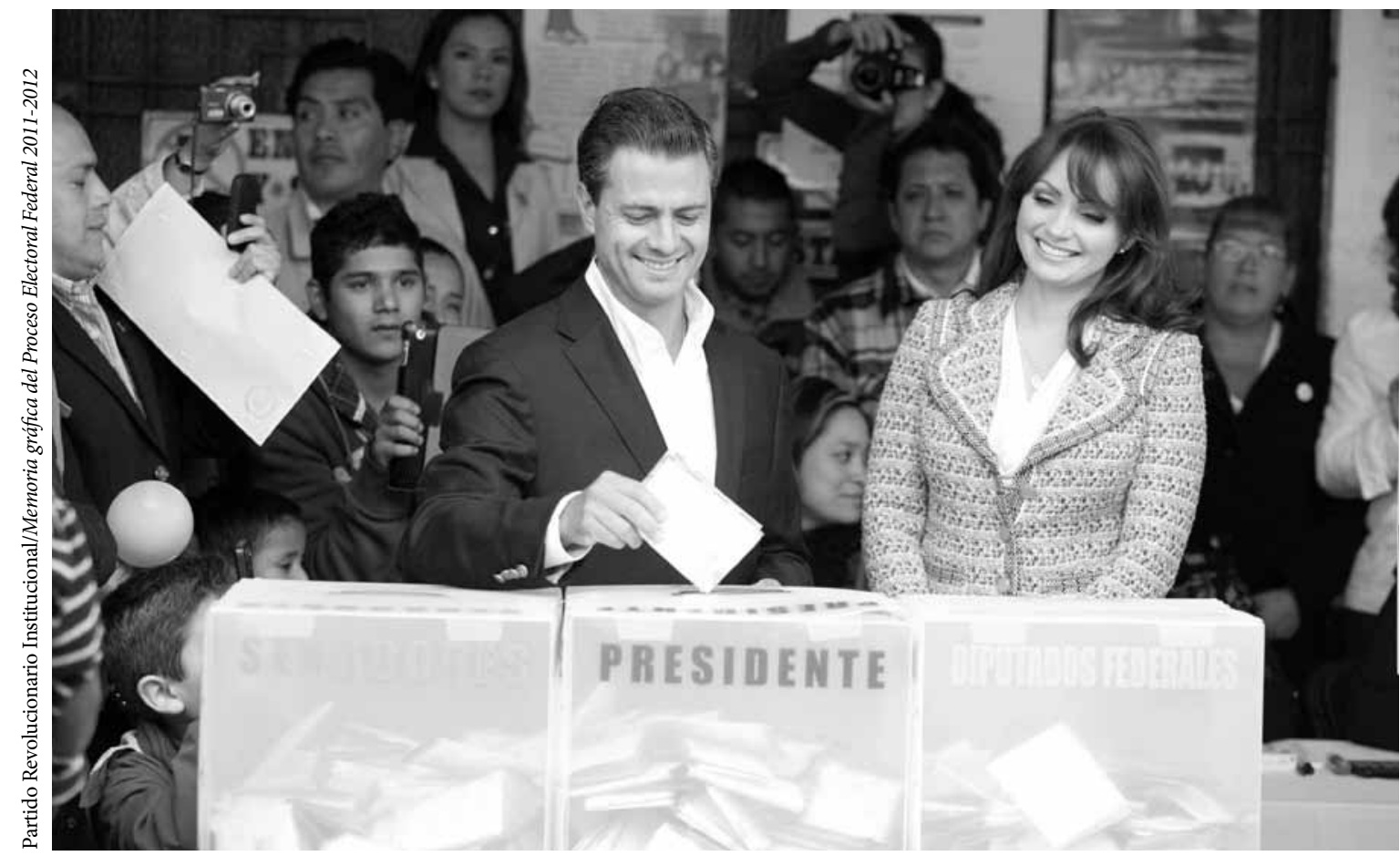

El candidato presidencial del Partido Revolucionario Institucional Enrique Peña Nieto vota en Atlacomulco, Estado de México, 2012.

conducir a la anulación del proceso. Así lo declaró el Trife al emitir su dictamen sobre las impugnaciones y quejas recibidas en torno a la calidad y legalidad de la elección. Varios miembros de \#YoSoy132 decidieron convertirse tardíamente en observadores después de comprobar que sus protestas contra las mentiras, la simulación y la corrupción de los medios de comunicación y de los poderes fácticos no cambiarían el triunfo del candidato impulsado por éstos. Entre varios ciudadanos quedó la percepción de que si bien todos los partidos incurrieron en faltas de apego a la ley y en el clientelismo, las instituciones electorales no hicieron lo suficiente para profundizar en la investigación de los incidentes que les fueron informados mediante quejas y denuncias ni actuaron de oficio ante la sospecha de falta de equidad en la contienda, por el interés y la prisa por declarar válida la elección.

Tres de los textos incluidos en este número de Desacatos hacen referencia a algunos aspectos que generaron la idea difundida sobre falta de equidad en la contienda y contribuyen con información, argumentos y dudas. Jesús Cantú, al analizar la información sobre los candidatos presidenciales difundida por los dos noticieros más vistos de Televisa y TVAzteca y por tres periódicos de circulación nacional: $E l$ Universal, La Jornada y Reforma, concluye que la igualdad política en la competencia electoral fue vulnerada por la intervención de los consorcios televisivos en favor de un candidato desde antes de iniciarse el proceso electoral. Al revisar el número de notas informativas, su duración, su tono y su distribución en el tiempo durante tres periodos desde el 1 de abril de 2011 hasta el 1 de julio del 2012, concluye que en la televisión no existe pluralismo interno ni externo y que seguramente ésta ejerce una gran influencia en la orientación del voto por el elevado porcentaje de mexicanos que se informa principalmente por este medio. De acuerdo con la "Encuesta Nacional de Cultura Política y Prácticas 


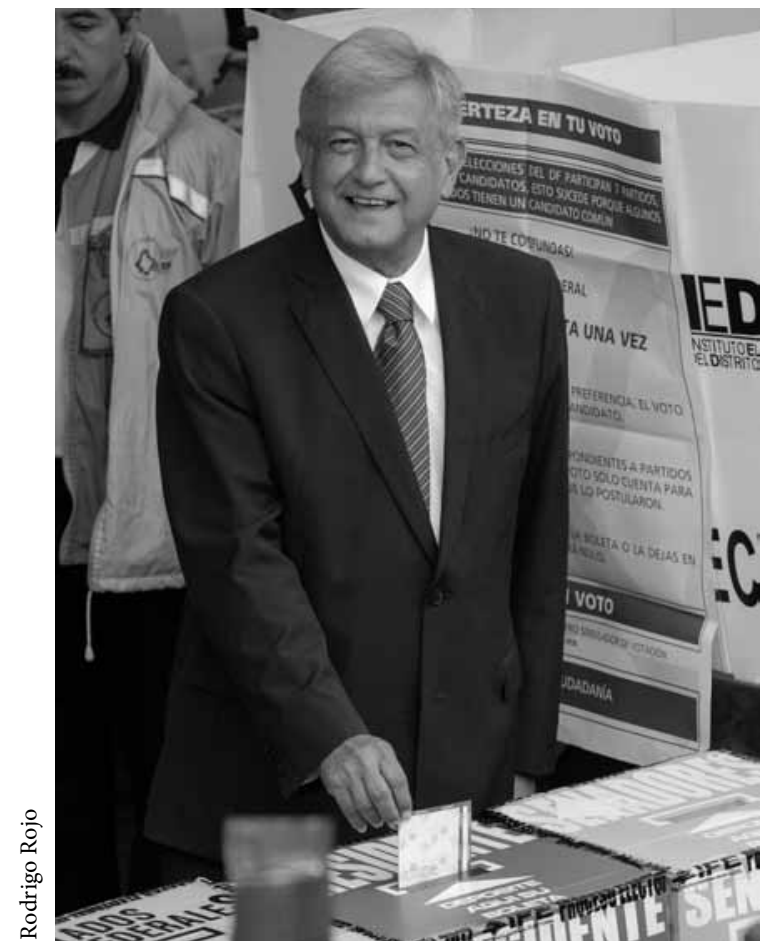

El candidato presidencial Andrés Manuel López Obrador ejerce su voto en esta contienda electoral, ciudad de México, 1 de julio 2012.

Ciudadanas 2012" (Segob, 2012) se trata de 76\% de los mexicanos. Respecto de los medios escritos observados detalla que si bien no existe pluralismo interno, sí se encuentra pluralismo externo al favorecer cada uno de ellos en mayor o menor medida a un candidato o a un partido. Sin embargo, según la misma encuesta, sólo $5.4 \%$ de la población tiene ese medio como su principal fuente de información. Con los datos que sustentan el análisis de este autor, aunque limitada a pocos medios de comunicación de gran influencia, hay evidencia de que la balanza llegó cargada a las elecciones en razón de la estrategia de los medios de comunicación, principalmente las televisoras, de colocar a Enrique Peña Nieto como el candidato presidencial que triunfaría.

Por su parte, José Antonio Crespo, en un minucioso análisis y con una acuciosa argumentación fundamentada en el análisis de información, busca mostrar por qué la izquierda no logra llegar al poder.
Desmenuza varias de las impugnaciones presentadas por el partido Movimiento Ciudadano (MC) con miras a la anulación de la elección. Señala las inconsistencias en las posiciones sostenidas en diferentes momentos por el MC y principalmente por su candidato Andrés Manuel López Obrador (AMLO). Así, mientras en un momento de la campaña AMLO alienta a los electores a aceptar los regalos y beneficios que les son ofrecidos a cambio de su voto, uno de los principales puntos de su demanda ante las instituciones electorales se relaciona con la compra e inducción del sufragio.

Con datos sobre la intención del voto y sobre la posición de los electores frente a los diferentes candidatos, Crespo descarta la posibilidad de que la diferencia de más de tres millones de votos entre López Obrador y Peña Nieto tenga su origen en una gran operación de compra de votos y en otras estrategias para alterar la voluntad de los electores. Muestra cómo el candidato de la izquierda llegó a la contienda con mayor rechazo de los electores - negativos - que apoyos - positivos - y cómo estaba obligado a remontar el alejamiento de los electores independientes que habían votado por él en 2006. Sostiene que muchos de ellos dejaron de apoyarlo por sus actos posteriores a la elección de ese año y por su desconocimiento de la institucionalidad, aun cuando los partidos de izquierda habían participado en su desarrollo.

Crespo cuestiona la estrategia de campaña de AMLO y en general de la izquierda, que se dirige fundamentalmente a sus votantes duros y aleja a los independientes más mesurados, menos radicales, menos anuentes al conflicto y también más volátiles. Está convencido de que la única manera de que la izquierda pueda llegar al poder radica en que sus estrategias de campaña logren suficientes votos de los electores independientes en niveles tales que le permitan superar la desventaja proporcional de votantes duros, comparada con los otros dos partidos. Para este autor lo ocurrido en la elección de 2012 respecto de la diferencia de votos entre el primero y segundo lugares se relaciona con una mala estrategia 
política de los partidos de izquierda, más que con el peso que pudieron tener las anomalías registradas y denunciadas por el Movimiento Ciudadano.

Cabría preguntarse si es posible pensar en elecciones equitativas, igualitarias y transparentes en México, en las que se permita a los ciudadanos elegir de manera libre e informada y asegurar que su voto será respetado, dada la profunda desigualdad existente en el país, el déficit de ciudadanía social y civil que padecen millones de mexicanos, el grado de concentración del poder económico y de los medios electrónicos de comunicación, la presencia de conspicuos poderes de hecho que se han colocado por encima y han capturado a los partidos y a los políticos, el manejo cupular y la ausencia de vida y prácticas democráticas en los partidos políticos y en varias organizaciones sociales y la presencia de corrupción diseminada y compartida.

Tres situaciones marcaron la elección pasada: el retorno del PRI al poder después de 12 años de gobiernos panistas de baja calidad democrática y elevados niveles de corrupción, el surgimiento espontáneo del movimiento de jóvenes universitarios \#YoSoy132 y la profunda crisis del Partido Acción Nacional tras haber estado en el poder. Considero que otra situación que se constató es la captura de los procesos electorales por las cúpulas de los partidos políticos y por los poderes de hecho, situación ampliamente denunciada por el Movimiento \#YoSoy132. Ni los militantes de los partidos políticos ni mucho menos las y los ciudadanos tuvieron intervención en la postulación de candidatos a la presidencia de la república ni al Congreso. Un número creciente de legisladores elegidos son representantes de intereses particulares de grupos económicos, de fracciones partidarias y, seguramente, del crimen organizado. Las elecciones tienen cada vez menos que ver con la vida y la decisión de las personas y son usadas para legitimar decisiones previamente tomadas por las elites para preservar sus intereses.

En un entorno tan poco propicio difícilmente lograremos elecciones en las que confluyan la legalidad y la legitimidad. Es cada vez más claro que no basta hacer cambios a la legislación y a las instituciones electorales para corregir vacíos y excesos cometidos en la elección previa. Ese modelo se ha agotado y resulta altamente costoso. Es necesario entrar a una revisión profunda de la institucionalidad en la que operan actualmente todos los agentes interesados en el acceso al poder, incluyendo al menos la legislación que regula la vida de los partidos políticos, las actividades políticas y de cabildeo de las empresas, las organizaciones y los gobiernos, las leyes relativas a la participación y organización ciudadana, las relacionadas con el crimen organizado, con las telecomunicaciones y con los servicios financieros en el país. Un aspecto central es rescatar la institucionalidad electoral en todo el país y ponerla en manos de ciudadanos y ciudadanas independientes que realicen una función de arbitraje profesional, equilibrada y equitativa. Asimismo, separar la atención a la de conflictividad entre los contendientes de la organización de los procesos a fin de garantizar igualdad en las contiendas. La creación de un Instituto Nacional de Elecciones como fue propuesto por el Comité Conciudadano para la Observación Electoral en 2007 (CCOE e Incide Social, 2007) parece una vía interesante para abatir, además, el costo de los procesos electorales.

\section{BIBLIOGRAFÍA}

Iniciativa Ciudadana y Desarrollo Social (Incide Social), 2006, Por un Congreso con rostro. ¿Qué representan los que quieren representarnos?, Iniciativa Ciudadana y Desarrollo Social, México.

Comité Conciudadano para la Observación Electoral (CCOE) e Iniciativa Ciudadana y Desarrollo Social (Incide Social), 2007, Propuesta ciudadana de reforma electoral, Iniciativa Ciudadana y Desarrollo Social, México.

, 2011, Observación de las instituciones electorales en el proceso 2008-2009. Consejo General del IFE y Tribunal Electoral del Poder Judicial, Comité Conciudadano para la Observación Electoral, Iniciativa Ciudadana y Desarrollo Social, México.

Secretaría de Gobernación (Segob), 2012, "Encuesta Nacional de Cultura Política y Prácticas Ciudadanas”, en línea: <http://www.encup.gob.mx/>. 